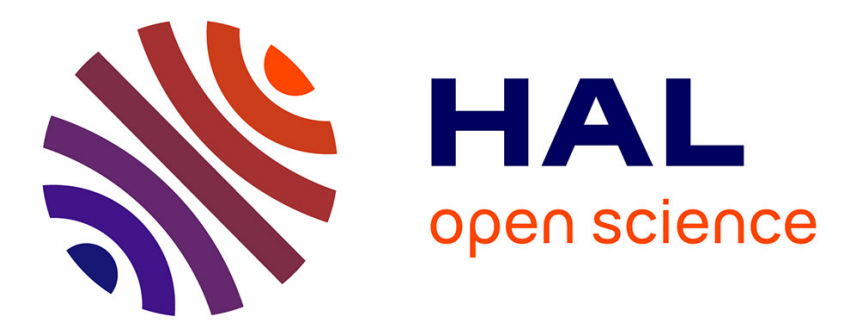

\title{
Narrowing power vs efficiency in synchronous set agreement
}

\author{
Achour Mostefaoui, Michel Raynal, Corentin Travers
}

\section{To cite this version:}

Achour Mostefaoui, Michel Raynal, Corentin Travers. Narrowing power vs efficiency in synchronous set agreement. [Research Report] PI 1836, 2007, pp.13. inria-00139286

\section{HAL Id: inria-00139286 \\ https://hal.inria.fr/inria-00139286}

Submitted on 30 Mar 2007

HAL is a multi-disciplinary open access archive for the deposit and dissemination of scientific research documents, whether they are published or not. The documents may come from teaching and research institutions in France or abroad, or from public or private research centers.
L'archive ouverte pluridisciplinaire HAL, est destinée au dépôt et à la diffusion de documents scientifiques de niveau recherche, publiés ou non, émanant des établissements d'enseignement et de recherche français ou étrangers, des laboratoires publics ou privés. 


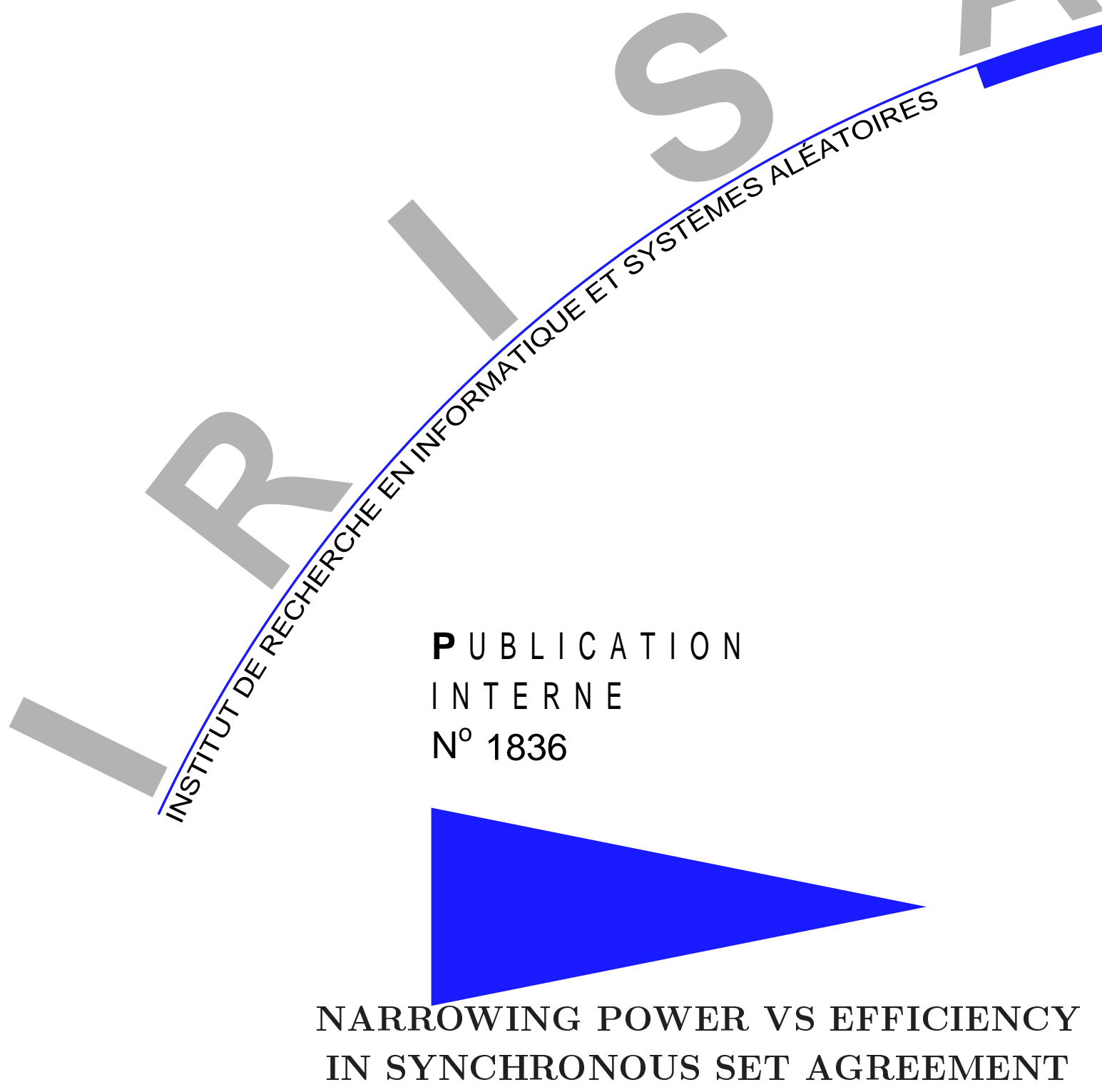

A. MOSTEFAOUI M. RAYNAL C. TRAVERS 



\title{
Narrowing power vs efficiency in synchronous set agreement
}

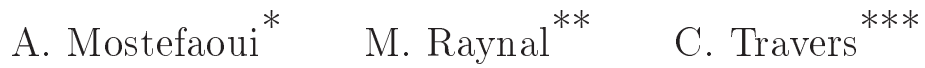 \\ Systèmes communicants \\ Projet ASAP
}

Publication interne $\mathrm{n}^{\circ} 1836$ - Mars 2007 - 13 pages

\begin{abstract}
The $k$-set agreement problem is a generalization of the uniform consensus problem: each process proposes a value, and each non-faulty process has to decide a value such that a decided value is a proposed value, and at most $k$ different values are decided. It has been shown that any algorithm that solves the $k$-set agreement problem in synchronous systems that can suffer up to $t$ crash failures requires $\left\lfloor\frac{t}{k}\right\rfloor+1$ rounds in the worst case. It has also been shown that it is possible to design early deciding algorithms where no process decides and halts after $\min \left(\left\lfloor\frac{f}{k}\right\rfloor+2,\left\lfloor\frac{t}{k}\right\rfloor+1\right)$ rounds, where $f$ is the number of actual crashes in a run $(0 \leq f \leq t)$.

This paper explores a new direction to solve the $k$-set agreement problem in a synchronous system. It considers that the system is enriched with base objects (denoted $[m, \ell]_{-}$SA objects) that allow solving the $\ell$-set agreement problem in a set of $m$ processes $(m<n)$. The paper has several contributions. It first proposes a synchronous $k$-set agreement algorithm that benefits from such underlying base objects. This algorithm requires $O\left(\frac{t \ell}{m k}\right)$ rounds, more precisely, $R_{t}=\left\lfloor\frac{t}{\Delta}\right\rfloor+1$ rounds, where $\Delta=m\left\lfloor\frac{k}{\ell}\right\rfloor+(k \bmod \ell)$. The paper then shows that this bound, that involves all the parameters that characterize both the problem $(k)$ and its environment $(t, m$ and $\ell$ ), is a lower bound. The proof of this lower bound sheds additional light on the deep connection between synchronous efficiency and asynchronous computability. Finally, the paper extends its investigation to the early deciding case. It presents a $k$-set agreement algorithm that directs the processes to decide and stop by round $R_{f}=\min \left(\left\lfloor\frac{f}{\Delta}\right\rfloor+2,\left\lfloor\frac{t}{\Delta}\right\rfloor+1\right)$. These bounds generalize the bounds previously established for solving the $k$-set problem in pure synchronous systems.
\end{abstract}

Key-words: Consensus, Efficiency, Lower bound, Round-based algorithm, Set agreement, Synchronous system, $t$-Resilience.

(Résumé : tsvp)

\footnotetext{
* IRISA, Université de Rennes 1, Campus de Beaulieu, 35042 Rennes Cedex, France achour@irisa.fr

** IRISA, Université de Rennes 1, Campus de Beaulieu, 35042 Rennes Cedex, France, raynal@irisa.fr

*** IRISA, Université de Rennes 1, Campus de Beaulieu, 35042 Rennes Cedex, France, travers@irisa.fr
}

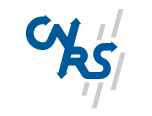

Centre National de la Recherche Scientifique (UMR 6074) Université de Rennes 1 - Insa de Rennes
Institut National de Recherche en Informatique et en Automatique - unité de recherche de Rennes 


\section{Puissance des objects de base vs efficacité pour l'accord ensembliste synchrone}

Résumé : Ce rapport étudie le lien entre la puissance des objects de base et l'efficacité dans les algorithmes d'accord ensembliste dans le cadre des systèmes synchrones.

Mots clés : Consensus, efficacité, borne inférieure, Ronde, Accord ensembliste, Système synchrone, Résilience. 


\section{Introduction}

Context of the work The $k$-set agreement problem generalizes the uniform consensus problem (that corresponds to the case $k=1$ ). That problem has been introduced by $\mathrm{S}$. Chaudhuri to investigate how the number of choices $(k)$ allowed to the processes is related to the maximum number $(t)$ of processes that can crash during a run [6]. The problem can be defined as follows. Each of the $n$ processors (processes) defining the system starts with a value (called a "proposed" value). Each process that does not crash has to decide a value (termination), in such a way that a decided value is a proposed value (validity), and no more than $k$ different values are decided (agreement) $)^{1}$.

When we consider asynchronous systems, the problem can trivially be solved when $k>t$. Differently, it has been shown that there is no solution in these systems as soon as $k \leq t[4,16,27]$. (The asynchronous consensus impossibility, case $k=1$, was demonstrated before, using a different technique $\left.[10]^{2}\right)$. Several approaches have been proposed to circumvent the impossibility to solve the $k$-set agreement problem in asynchronous systems (e.g., probabilistic protocols [24], unreliable failure detectors with limited scope accuracy [14, 23], or conditions associated with input vectors [21]).

The situation is different in synchronous systems where the $k$-set agreement problem can always be solved, whatever the respective values of $t$ and $k$. This has an inherent cost, namely, the smallest number of rounds (time complexity measured in communication steps) that have to be executed in the worst case scenario is lower bounded by $\left\lfloor\frac{t}{k}\right\rfloor+1[7]$. (That bound generalizes the $t+1$ lower bound associated with the consensus problem $[1,3,9,19]$.)

Although failures do occur, they are rare in practice. For the uniform consensus problem $(k=1)$, this observation has motivated the design of early deciding synchronous protocols [8, 18, 25], i.e., protocols that can cope with up to $t$ process crashes, but decide in less than $t+1$ rounds in favorable circumstances (i.e., when there are few failures). More precisely, these protocols allow the processes to decide in $\min (f+2, t+1)$ rounds, where $f$ is the number of processes that crash during a run, $0 \leq f \leq t$, which has been shown to be optimal (the worst scenario being when there is exactly one crash per round) [5, 17, 29].

In a very interesting way, it has also been shown that the early deciding lower bound for the $k$-set agreement problem is $\min \left(\left\lfloor\frac{f}{k}\right\rfloor+2,\left\lfloor\frac{t}{k}\right\rfloor+1\right)[12]$. This lower bound, not only generalizes the corresponding uniform consensus lower bound, but also shows an "inescapable tradeoff" among the number $t$ of faults tolerated, the number $f$ of actual faults, the degree $k$ of coordination we want to achieve, and the best running time achievable. It is important to notice that, when compared to consensus, $k$-set agreement divides the running time by $k$ (e.g., allowing two values to be decided halves the running time).

Related work To our knowledge, two approaches have been proposed and investigated to circumvent the $\min \left(\left\lfloor\frac{f}{k}\right\rfloor+2,\left\lfloor\frac{t}{k}\right\rfloor+1\right)$ lower bound associated with the synchronous $k$-set agreement problem.

The first is the fast failure detector approach that has been proposed and developed in [2] to expedite decision in synchronous consensus. That approach assumes a special hardware that allows a process to detect the crash of any process at most $d$ time units after the crash occurred, where $d<D, D$ being the maximum message delay provided by the synchronous system. Both $d$ and $D$ are a priori known by the processes. A fast failure detector-based consensus algorithm that terminates in $D+f d$ is proposed in [2], where it is also shown that $D+f d$ is a lower bound for any algorithm based on a fast failure detector ${ }^{3}$. To our knowledge, this approach has been considered only for the consensus problem.

A second approach that has been proposed to circumvent the $\min (f+2, t+1)$ lower bound is the use of conditions [22]. That approach considers that the values proposed by the processes define an input vector with one entry per process. Basically, a condition $C_{t}^{d}(t$ and $d$ are two parameters that allow defining instances of the condition) is a set of input vectors $I$ such that $\forall I \in C_{t}^{d}$, there is a value that appears in $I$ more than $t-d$ times. A deterministic way to define which value has to appear enough times in a

\footnotetext{
${ }^{1}$ This paper considers the crash failure model. The reader interested by the $k$-set agreement problem in more severe send/receive/general omission failure models can consult the introductory survey [26].

${ }^{2}$ The impossibility to solve consensus in asynchronous systems is usually named "FLP result" according to the names of its authors [10].

${ }^{3}$ Without a fast failure detector, the cost would be $D \times \min (f+2, t+1)$.
}

PI ${ }^{\circ} 1836$ 
vector $I$ (e.g., the maximal value of the vector [20]) allows defining a hierarchy of conditions such that $C_{t}^{0} \subset \cdots \subset C_{t}^{x} \subset \cdots \subset C_{t}^{t}$ (where $C_{t}^{t}$ is the condition including all the input vectors).

[22] presents two main results. Let $I$ be the input vector of the considered run, and $C_{t}^{d}$ be a condition. The first result is a synchronous consensus algorithm that allows the processes to decide in (1) one round when $I \in C_{t}^{d}$ and $f=0,(2)$ two rounds when $I \in C_{t}^{d}$ and $f \leq t-d,(3) \min (d+1, f+2, t+1)$ rounds when $I \in C_{t}^{d}$ and $f>t-d$, and $(4) \min (f+2, t+1)$ when $I \notin C_{t}^{d}$. The second result is a proof showing that $\min (d+1, f+2, t+1)$ rounds are necessary in the worst case when $I \in C_{t}^{d}$ (and $I \notin C_{t}^{d-1}$ ).

An extension of this condition-based approach (combined with the use of appropriate failure detectors) to solve the $k$-set agreement problem in asynchronous systems has been considered in [21]. It is shown that $k>d$ is a necessary and sufficient requirement for obtaining an asynchronous $k$-set agreement algorithm based on a condition $C_{t}^{d}$.

Problem addressed in the paper The paper is on the efficiency (measured as the number of rounds required to decide) of synchronous set agreement algorithms. As it has just been shown, fast failure detectors and conditions are two ways to circumvent the synchronous lower bound. The paper investigates a third approach. That approach is based on base objects that allow narrowing the set of proposed values. Their aim is to play a part similar to fast failure detectors or conditions, i.e., allow expediting consensus.

Let us consider as a simple example a test\&set object. This object has consensus number 2 [13], which means that it allows solving consensus in an asynchronous system made up of two processes (where one of them can crash), but not in a system made up of $n>2$ processes (where up to $n-1$ can crash) ${ }^{4}$. Is it possible to use such base objects to speed up synchronous set agreement in a system made up of $n$ processes where up to $t$ may crash? More generally, let $[m, \ell]$ SA denote an object that allows solving $\ell$-set agreement in a synchronous system of $m$ processes. As fast failure detectors or conditions, these objects are assumed given for free. So, the previous question becomes:

- Is it possible to benefit from [m, $\ell]_{-S A}$ objects to build a $t$-resilient synchronous $[n, k]$ _SA object (i.e., a $k$-set agreement object that has to cope with up to $t$ process crashes)?

- If such a construction is possible, is its cost smaller than $\left\lfloor\frac{t}{k}\right\rfloor+1$, or smaller than $\min \left(\left\lfloor\frac{f}{k}\right\rfloor+2,\left\lfloor\frac{t}{k}\right\rfloor+1\right)$ if we are interested in an early deciding $[n, k] \_$SA object?

If $m, \ell, n$ and $k$ are such that there is an integer $a$ with $n \leq a m$ and $a \ell \leq k$, it is possible to solve the $k$-set agreement problem without exchanging any value (i.e., in 0 round!) whatever the value of $t$. This is trivially obtained by partitioning the $n$ processes into $a$ subsets of at most $m$ processes, and using in each subset a $[m, \ell] S A$ object in order that each process be provided with a decided value. So, the interesting cases are when the values $m, \ell, n$ and $k$ do not allow a trivial partitioning such as the previous one.

Another way to present the previous question is the following: how much crashes can we tolerate when we want to build a synchronous [10,3]_SA object from [2,1]_SA objects, if one wants to decide in at most one round? In at most two rounds? In at most three rounds?

From a more practical point of view, we can see the system as made up of clusters of $m$ processes, such that an operation involving only processes of a given cluster can be performed very efficiently, i.e., in a time that is smaller than the maximal message transfer delay involving processes belonging to different clusters. That is the sense in which the sentence "the $[m, \ell]$ _SA objects are given for free" has to be understood.

Results The paper presents the following results.

- It first presents a synchronous message-passing algorithm that builds a $[n, k]$ SA object from $[m, \ell]$ _SA objects. This algorithm works for any values of $n, k, m$, and $\ell$ (assuming, of course, $n>k$ and $m>\ell$ ).

- The paper then shows that the number of rounds $\left(R_{t}\right)$ of the previous algorithm varies as $O\left(\frac{t \ell}{m k}\right)$. This means that $R_{t}$ (1) decreases when the coordination degree $k$ increases (i.e., when less synchronization is required), or when the number of processes $m$ involved in each underlying object increases, and

\footnotetext{
${ }^{4}$ The consensus number of a concurrent object type is the maximum number of processes that can solve consensus (despite any number of process crashes) using only atomic registers and objects of that type. The consensus number of test\&set objects, queues, and stacks is 2 [13].
} 
(2) increases when the underlying object is less and less powerful (i.e., when $\ell$ increases) or when the number of process crashes that the algorithm has to tolerate increases. More precisely, we have:

$$
R_{t}=\left\lfloor\frac{t}{m\left\lfloor\frac{k}{\ell}\right\rfloor+(k \bmod \ell)}\right\rfloor+1 .
$$

When we consider the previous example of building, in a synchronous system, a [10,3]_SA object from $[2,1]$ SA objects, we can conclude that $R_{t}=1$ requires $t<6$, while $R_{t}=2$ allows $t=9$. Moreover, as there are only $n=10$ processes, there is no value of $t$ that can entail an execution in which $R_{t}=3$ are required (for it to occur, we should have $12 \leq t<18$ and $n>t$ ).

To have a better view of $R_{t}$, it is interesting to look at special cases.

- Case 1. Build a consensus object in a synchronous system from $[1,1]$ SA base objects or $[m, m]$ SA objects (i.e., from base objects that have no power). It is easy to see that $R_{t}=t+1$ (that is the well-known lower bound for synchronous $t$-resilient consensus).

- Case 2. Build a $[n, k]$ _SA object in a synchronous system from $[1,1]$ _SA base objects or $[m, m]$ SA objects (base objects without power). It is easy to see that $R_{t}=\left\lfloor\frac{t}{k}\right\rfloor+1$, (that is the lower bound for synchronous $t$-resilient $k$-set agreement).

- Case 3. Build a synchronous consensus from [m,1]_SA base objects (i.e., consensus objects). In that case $R_{t}=\left\lfloor\frac{t}{m}\right\rfloor+1$.

- Case 4. Build a synchronous $[n, \ell]_{-}$SA object from $[m, \ell]$ SA base objects. In that case, $R_{t}=$ $\left\lfloor\frac{t}{m}\right\rfloor+1$.

- Case 5. Build a synchronous [n, $k]$ _SA object from [ $m, 1]$ _SA base objects (i.e., consensus objects). We then have $R_{t}=\left\lfloor\frac{t}{m k}\right\rfloor+1$.

These particular instances show clearly how the coordination degree and the size of the base objects (measured by the value $m$ ) affect the maximal number of rounds executed by the algorithm and consequently allow expediting the decision.

- The paper then shows that the value $R_{t}$ is optimal when, one wants to build, in a synchronous system, an $[n, k]$ SA object from $[m, \ell] \_S A$ base objects. This optimality result generalizes previous lower bounds proved for special cases such as consensus [1, 9, 18], and set agreement [7].

The optimality proof relies on two theorems, one from Gafni [11], the other from Herlihy and Rajsbaum [15]. Gafni's theorem establishes a deep connection between solvability in asynchronous system and lower bounds (efficiency) in synchronous systems. Herlihy and Rajsbaum's theorem is on the impossibility to solve some set agreement problems in asynchronous systems.

- Finally, the paper extends the algorithm to the early decision case. More specifically, the maximal number of rounds of the early deciding version of the algorithm is the following:

$$
R_{f}=\min \left(\left\lfloor\frac{f}{\Delta}\right\rfloor+2,\left\lfloor\frac{t}{\Delta}\right\rfloor+1\right) \quad \text { where } \quad \Delta=m\left\lfloor\frac{k}{\ell}\right\rfloor+(k \bmod \ell) .
$$

It is easy to see that this early decision bound generalizes the lower bounds that are known for the special consensus and set agreement cases.

This paper is an endeavor to capture the essence of the synchronous set agreement and provide the reader with a better understanding of it. To that end, it considers design simplicity as a first-class citizen when both designing algorithms and proving lower bound results ${ }^{5}$.

As already noticed, the lower bound proof relies on previous theorems. We do think that Gafni's theorem [11] (that states that an asynchronous system with at most $t^{\prime}$ crashes can implement the first $\left\lfloor\frac{t}{t^{\prime}}\right\rfloor$ rounds of a synchronous system with up to $t$ failures) is a fundamental theorem of fault-tolerant distributed computing. The lower bound proof of this paper paper shows an application of this powerful theorem.

\footnotetext{
${ }^{5}$ The paper strives to modestly follow Einstein's advice "Make it as simple as possible, but no more". PI ${ }^{\circ} 1836$
} 
Roadmap The paper is made up of 6 sections. Section 2 introduces the system model and definitions. Section 3 presents the algorithm that builds an $[n, k]$ _SA object from $[m, \ell]$ _SA objects in $R_{t}$ synchronous rounds. Section 4 proves that $R_{t}$ is a lower bound on the number of rounds for any synchronous algorithm that builds an $[n, k]$ SA object from $[m, \ell] \_$SA objects. Section 5 considers the early decision case. Finally, 6 concludes the paper.

\section{Computation model and the set agreement problem}

The $k$-set agreement problem The problem has been informally stated in the Introduction: every process $p_{i}$ proposes a value $v_{i}$ and each correct process has to decide on a value in relation to the set of proposed values. More precisely, the $k$-set agreement problem [6] is defined by the following three properties (as we can see 1-set agreement is the uniform consensus problem):

- Termination: Every correct process eventually decides.

- Validity: If a process decides $v$, then $v$ was proposed by some process.

- Agreement: No more than $k$ different values are decided.

Process model The system model consists of a finite set of $n$ processes, namely, $\Pi=\left\{p_{1}, \ldots, p_{n}\right\}$. A process is a sequence of steps (execution of a base atomic operation). A process is faulty during an execution if it stops executing steps (after it has crashed a process executes no step). As already indicated, $t$ is an upper bound on the number of faulty processes, while $f$ denotes the number of processes that crash during a particular run, $0 \leq f \leq t<n$. (Without loss of generality we consider that the execution of a step by a process takes no time.)

In the following, we implicitly assume $k \leq t$. This is because $k$-set agreement can trivially be solved in synchronous or asynchronous systems when $t<k[6]$.

Communication/coordination model The processes communicate by sending and receiving messages through channels. Every pair of processes $p_{i}$ and $p_{j}$ is connected by a channel. The sending of a message and the reception of a message are atomic operations. The underlying communication system is assumed to be failure-free: there is no creation, alteration, loss or duplication of message.

In addition to messages, the processes can coordinate by accessing $[m, \ell]$ SA objects. Such an object is a one-shot object that can be accessed by at most $m$ processes. Its power is to solve the $\ell$-set agreement problem among $m$ processes. Let us observe that, for $1 \leq m \leq n$, an $[m, m]$ SA object is a trivial object that has no coordination power.

Round-based synchrony The system is synchronous. This means that each of its runs consists of a sequence of rounds. Those are identified by the successive integers 1,2, etc. For the processes, the current round number appears as a global variable $r$ that they can read, and whose progress is given for free: it is managed by an external entity. A round is made up of two main consecutive phases:

- A send phase in which each process sends zero or one message to each other processes ${ }^{6}$. If a process crashes during the send phase of a round, an arbitrary subset of the processes to which it sent messages will receive these messages.

- A receive phase in which each process receives messages. The fundamental property of the synchronous model lies in the fact that a message sent by a process $p_{i}$ to a process $p_{j}$ at round $r$, is received by $p_{j}$ at the very same round $r$.

Before or after a phase, a process can execute local computations (e.g., process the messages it received during the current round). It can also invokes an underlying $[m, \ell]$ SA base object.

\footnotetext{
${ }^{6}$ It is easy to see that this model has the same power as the model where, at each round, each process has to send the same message to all the processes $[3,19,28]$.
} 


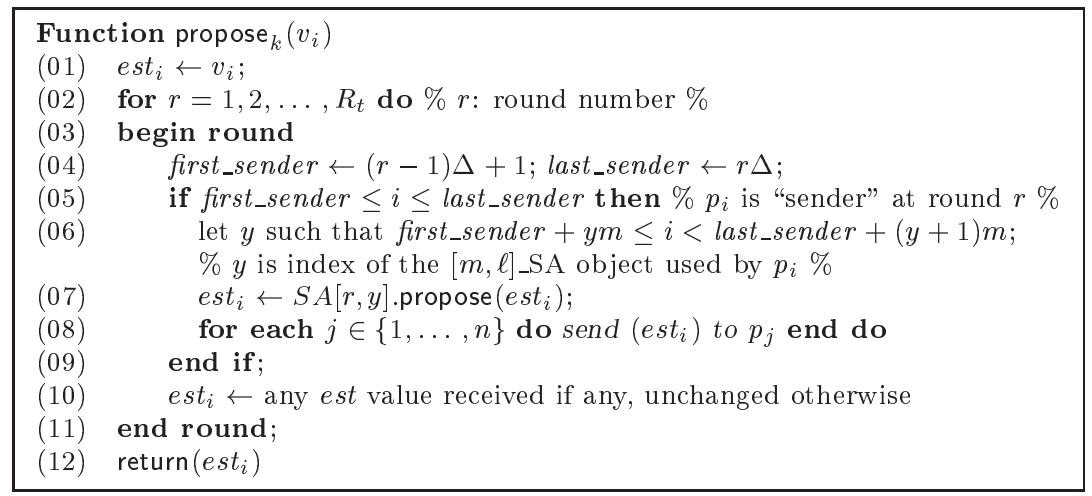

Figure 1: $[n, k]$ SA object from $[m, \ell]$ SA objects in a synchronous system (code for $p_{i}$ )

\section{A synchronous $[n, k]$ SA algorithm}

This section presents a simple algorithm that, when at most $t$ processes may crash, builds an $[n, k]$ _SA object if the system provides the $n$ processes with round-based synchrony and $[m, \ell] \_S A$ base objects.

Notation In all the rest of the paper we are using the following notations:

- $k=\alpha \ell+\beta$ with $\alpha=\left\lfloor\frac{k}{\ell}\right\rfloor$ and $\beta=k \bmod \ell$.

- $\Delta=\alpha m+\beta$ and $R_{t}=\left\lfloor\frac{t}{\Delta}\right\rfloor+1=\left\lfloor\frac{t}{m\left\lfloor\frac{k}{\ell}\right\rfloor+(k \bmod \ell)}\right\rfloor+1$.

\subsection{The algorithm}

The algorithm is pretty simple. It is described in Figure 1. A process $p_{i}$ invokes the operation propose ${ }_{k}\left(v_{i}\right)$ where $v_{i}$ is the value it proposes. That value is initially stored in the local variable est $t_{i}$ (line 01), that afterwards will contain the current estimate of $p_{i}$ 's decision value (line 10). The process terminates when it executes the return $\left(e s t_{i}\right)$ statement.

Each process executes $R_{t}$ rounds (line 02 ). During any round $r$, only $\Delta$ processes are allowed to send their current estimates. These processes are called the senders of round $r$. When $r=1$, they are the processes $p_{1}, \ldots, p_{\Delta}$, during the second round the processes $p_{\Delta+1}, \ldots, p_{2 \Delta}$, and so on (lines 04-05).

The $\Delta$ senders of a round $r$ are partitioned into $\left\lceil\frac{\Delta}{m}\right\rceil$ subsets of $m$ processes (the last subset containing possibly less than $m$ processes), and each subset uses an $[m, \ell]$ SSA object to narrow the set of its current estimates (lines 06-07). After this "narrowing", each sender process sends its new current estimate to all the processes. A process $p_{i}$ accesses an $[m, \ell]$ SA object by invoking the operation propose $\left(e s t_{i}\right)$. The $\left\lceil\frac{\Delta}{m}\right\rceil$ $[m, \ell]$ SA objects used during a round $r$ are in the array $S A\left[r, 0 . .\left\lceil\frac{\Delta}{m}\right\rceil-1\right]^{7}$. Finally, when during a round, a process $p_{i}$ receives estimates, it updates est ${ }_{i}$ accordingly (line 10).

It is important to see that, if during a round, at least one sender process does not crash, at most $k=\alpha \ell+\beta$ estimates are sent during that round, which means that $k$-set agreement is guaranteed as soon as there is a round during which an active process does not crash.

\subsection{Proof of the algorithm}

Lemma 1 Let $n c[r]$ be the number of processes that crash during the round $r$. There is a round $r$ such that $r \leq R_{t}$ and $n c[r]<\Delta$.

\footnotetext{
${ }^{7}$ Actually, only $R_{t}\left\lfloor\frac{\Delta}{m}\right\rfloor$ base $[m, \ell] \_$SA objects are needed. This follows from the following observation: during each round $r$, if $\beta \neq 0$, the "last" $\beta$ sender processes do not need to use such an $[m, \ell]$ _SA object because $\beta \leq \ell$. (Let us recall that $0 \leq \beta<\ell$ and $\Delta$ is defined as $\alpha m+\beta$.)
} 
Proof Let $t=\alpha^{\prime} \Delta+\beta^{\prime}$ with $\alpha^{\prime}=\left\lfloor\frac{t}{\Delta}\right\rfloor$ and $\beta^{\prime}=t \bmod \Delta$. The proof is by contradiction. let us assume that, $\forall r \leq R_{t}$, we have $n c[r] \geq \Delta$. We then have:

$$
\sum_{r=1}^{R_{t}} n c[r] \geq \Delta \times R_{t}=\Delta\left(\left\lfloor\frac{t}{\Delta}\right\rfloor+1\right)=\Delta\left(\alpha^{\prime}+\left\lfloor\frac{\beta^{\prime}}{\Delta}\right\rfloor+1\right)=\Delta \times \alpha^{\prime}+\Delta>t .
$$

Consequently, there are more than $t$ processes that crash: a contradiction.

Lemma 2 At any round $r$, at most $k$ different estimate values are sent by the processes.

Proof Let us recall that $k=\alpha \ell+\beta$ (Euclidean division of $k$ by $\ell$ ) and the value $\Delta$ is $\alpha m+\beta$.

Due to the lines 04-05, at most $\Delta$ processes are sender at each round $r$. These $\Delta$ sender processes are partitioned into $\left\lfloor\frac{\Delta}{m}\right\rfloor$ sets of exactly $m$ processes plus a set of $\beta$ processes. As each underlying [m, $\ell$ ]SA object used during the round $r$ outputs at most $\ell$ estimates values from the value it is proposed, it follows that at most $\alpha \ell+\beta$ estimates values can be output by these objects, which proves the lemma. $\square_{\text {Lemma } 2}$

Lemma 3 At most $k$ different values are decided by the processes.

Proof At any round the number of senders is at most $\Delta$ (lines 04-05). Moreover, due to lemma 1, there is at least one round $r \leq R_{t}$ during which a correct process is a sender. If follows from Lemma 2, line 08 and line 10, that, at the end of such a round $r$, the estimates of the processes contain at most $k$ distinct values.

Theorem 1 The algorithm described in Figure 1 is a synchronous t-resilient $k$-set agreement algorithm.

Proof The termination property follows directly from the synchrony of the model: a process that does not crash executes $R_{t}$ rounds. The validity property follows directly from the initialization of the estimate values $e t_{i}$, the correctness of the underlying $[m, \ell]$ SA objects (line 07 ), and the fact that the algorithm exchanges only est $t_{i}$ values. Finally, the agreement property is Lemma 3.

$\square_{\text {Theorem } 1}$

\section{Lower bound on the number of rounds}

This section proves that the previous algorithm is optimal with respect to the number of rounds. The proof of this lower bound is based on (1) a deep connection relating synchronous efficiency and asynchronous computability in presence of failures [11], and (2) an impossibility result in asynchronous set agreement [15].

\subsection{Notation and previous results}

This section uses the following notations.

- $\mathcal{S}_{n, t}[\emptyset]$ denotes the classical round-based synchronous system model made up of $n$ processes, where up to $t$ processes may crash $[3,19,28]$.

- $\mathcal{S}_{n, t}[m, \ell]$ is the $\mathcal{S}_{n, t}[\emptyset]$ system model enriched with $[m, \ell] S$ SA objects. This is the model defined in Section 2 ( $n$ processes, at most $t$ process crashes, coordination possible through $[m, \ell] S A$ objects).

- $\mathcal{A S}_{n, t}[\emptyset]$ denotes the classical asynchronous system model (n processes, up to processes $t$ may crash, no additional equipment).

- $\mathcal{A S}_{n, t}[m, \ell]$ denotes the asynchronous system model $\mathcal{A S}_{n, t}[\emptyset]$ enriched with $[m, \ell] \_$SA objects. (From a computability point of view, $\mathcal{A S}_{n, t}[\emptyset]$ is weaker than $\mathcal{A S}_{n, t}[m, \ell]$.)

The following theorems are central in proving that $R_{t}$ is a lower bound. 
Theorem 2 (Gafni [11]) Let $n>t \geq k>0$. It is possible to simulate in $\mathcal{A S}_{n, k}[\emptyset]$ the first $\left\lfloor\frac{t}{k}\right\rfloor$ rounds of any algorithm designed for $\mathcal{S}_{n, t}[\emptyset]$ system model.

The next corollary is a simple extension of Gafni's theorem suited to our needs.

Corollary 1 Let $n>t \geq k>0$. It is possible to simulate in $\mathcal{A S}_{n, k}[m, \ell]$ the first $\left\lfloor\frac{t}{k}\right\rfloor$ rounds of any algorithm designed for $\mathcal{S}_{n, t}[\mathrm{~m}, \ell]$ system model.

Theorem 3 (Herlihy-Rajsbaum [15]) Let $J_{m, \ell}$ be the function $u \rightarrow \ell\left\lfloor\frac{u}{m}\right\rfloor+\min (\ell, u \bmod m)-1$. There is no algorithm that solves the $K$-set agreement problem, with $K=J_{m, \ell}(t+1)$, in $\mathcal{A S}_{n, t}[m, \ell]$.

\subsection{The lower bound}

Theorem 4 Let $1 \leq \ell \leq m<n$ and $1 \leq k \leq t<n$. Any algorithm that solves the $k$-set agreement problem in $\mathcal{S}_{n, t}[m, \ell]$ has at least one run in which at least one process does not decide before the round $R_{t}=\left\lfloor\frac{t}{m\left\lfloor\frac{k}{\ell}\right\rfloor+(k \bmod \ell)}\right\rfloor+1$.

Proof The proof is by contradiction. let us assume that there is an algorithm $A$ that solves the $k$-set agreement problem in at most $R<R_{t}$ rounds in $\mathcal{S}_{n, t}[m, \ell]$ (this means that any process decides by at most $R$ rounds, or crashes before). We consider two cases.

- $k<\ell$. We have then $R<R_{t}=\left\lfloor\frac{t}{k}\right\rfloor+1$.

1. As $k<\ell$, the $\ell$-set agreement can be solved in in $\mathcal{A S}_{n, k}[\emptyset]$. It follows that, as far as set agreement is concerned, $\mathcal{A S}_{n, k}[\emptyset]$ and $\mathcal{A S}_{n, k}[m, \ell]$ have the same computational power.

2. It follows from the corollary of Gafni's theorem that there is, in $\mathcal{A S}_{n, k}[m, \ell]$, a simulation of the first $\left\lfloor\frac{t}{k}\right\rfloor$ rounds of any algorithm designed for the $\mathcal{S}_{n, t}[m, \ell]$ system model. It is consequently possible to simulate in $\mathcal{A S}_{n, k}[m, \ell]$ the $R<R_{t}=\left\lfloor\frac{t}{k}\right\rfloor+1$ rounds of the algorithm $A$. It follows that the $k$-set agreement problem can be solved in in $\mathcal{A S}_{n, k}[m, \ell]$.

3. Combining the two previous items, we obtain an algorithm that solves the $k$-set agreement problem in $\mathcal{A S}_{n, k}[\emptyset]$. This contradicts the impossibility to solve the $k$-set agreement problem in $\mathcal{A S}_{n, k}[\emptyset]$ $[4,16,27]$. This proves the theorem for the case $k<\ell$.

- $k \geq \ell$. Let us recall the definition $\Delta=m\left\lfloor\frac{k}{\ell}\right\rfloor+(k \bmod \ell)=\alpha m+\beta$.

1. It follows from the corollary of Gafni's theorem that at least $\left\lfloor\frac{t}{\Delta}\right\rfloor$ rounds of any algorithm designed for the $\mathcal{S}_{n, t}[m, \ell]$ system model can be simulated in $\mathcal{A S}_{n, \Delta}[m, \ell]$.

So, as the algorithm $A$ solves the $k$-set agreement problem in $\mathcal{S}_{n, t}[m, \ell]$, in at most $R<R_{t}=$ $\left\lfloor\frac{t}{\Delta}\right\rfloor+1$, combining the simulation with $A$, we obtain an algorithm that solves the $k$-set agreement problem in $\mathcal{A S}_{n, \Delta}[m, \ell]$.

2. Considering the argument used in Herlihy-Rajsbaum's theorem we have the following:

$$
\begin{aligned}
J_{m, \ell}(\Delta+1) & =\ell\left\lfloor\frac{\Delta+1}{m}\right\rfloor+\min (\ell,(\Delta+1) \bmod m)-1, \\
& =\ell\left\lfloor\frac{\alpha m+\beta+1}{m}\right\rfloor+\min (\ell,(\alpha m+\beta+1) \bmod m)-1, \\
& =\ell\left(\alpha+\left\lfloor\frac{\beta+1}{m}\right\rfloor\right)+\min (\ell,(\beta+1) \bmod m)-1 .
\end{aligned}
$$

Let us observe that $\ell \leq m$. Moreover, as $\beta=k \bmod \ell$, we also have $\beta<\ell$. To summarize: $\beta<\ell \leq m$. There are two cases to consider.

(a) $m=\beta+1$. Observe that this implies that $\ell=m$ and $\ell-1=\beta$.

$$
\begin{aligned}
J_{m, \ell}(\Delta+1) & =\ell(\alpha+1)+\min (\ell, m \bmod m)-1, \\
& =\ell \alpha+\ell-1=\ell \alpha+\beta=k .
\end{aligned}
$$

PI ${ }^{\circ} 1836$ 
(b) $m>\beta+1$ :

$$
\begin{aligned}
J_{m, \ell}(\Delta+1) & =\ell \alpha+\min (\ell,(\beta+1) \bmod m)-1, \\
& =\ell \alpha+\beta+1-1=k .
\end{aligned}
$$

In both cases, $J_{m, \ell}(\Delta+1)=k$. It follows from Herlihy-Rajsbaum's theorem that there is no algorithm that solves the $J_{m, \ell}(\Delta+1)$-set agreement problem (i.e., the $k$-set agreement problem) in $\mathcal{A S}_{n, \Delta}[m, \ell]$.

3. The two previous items contradict each other, thereby proving the theorem for the case $k<\ell$.

Corollary 2 When $k<\ell$, the underlying $[m, \ell] \_S A$ objects are useless.

Proof The corollary follows from the fact that $k<\ell \Rightarrow R_{t}=\left\lfloor\frac{t}{k}\right\rfloor+1$, that is the lower bound when no underlying base object is used.

$\square_{\text {Corollary } 2}$

This corollary means that no $k$-set agreement algorithm can benefit from $[m, \ell]$ SA objects when $k<\ell$.

\section{Early decision}

This section extends the algorithm described in Figure 1 in order to obtain an early-deciding algorithm. As announced in the Introduction, the resulting algorithm allows the processes to decide by round $R_{f}=$ $\min \left(\left\lfloor\frac{f}{\Delta}\right\rfloor+2,\left\lfloor\frac{t}{\Delta}\right\rfloor+1\right)$, where $\Delta=m\left\lfloor\frac{k}{\ell}\right\rfloor+(k \bmod \ell)$.

\subsection{The early deciding algorithm}

This algorithm is described in Figure 2. It is obtained from the base algorithm in a surprisingly simple way: only two new statements are added to the base algorithm to obtain early decision. These are the new lines, named A1 and A2, inserted between line 09 and line 10. No statement of the base algorithm has to be modified or suppressed.

The design principles of this algorithm are very simple. A process $p_{i}$ that is a sender during a round $r^{\prime}$ and participates in the next round $r^{\prime}+1$ (so, it has not crashed by the end of $r^{\prime}$ ), sends to all the processes a control message (denoted COMMIT) during the round $r^{\prime}+1$ (additional line A1). In that way, $p_{i}$ informs all the processes that the estimate value it sent during the previous round $r^{\prime}$ was received by all the processes (this follows from the communication synchrony property). Moreover, as at most $k$ different values are sent during a round (Lemma 2), and at least one process (namely, $p_{i}$ ) sent a value to all during $r^{\prime}$, it follows from the fact that $p_{i}$ participates to the round $r^{\prime}+1$ that the estimates of all the processes contain at most $k$ different values at the end of $r^{\prime}$. Consequently, a process that receives a COMMIT message during a round $r^{\prime}+1$ can decide the value of its estimate at the end of the round $r^{\prime}$ and stops (additional line A2).

It is easy to see that if at least one process in $p_{1}, \ldots, p_{\Delta}$ does not crash, the processes decide in two rounds. If all the processes $p_{1}, \ldots, p_{\Delta}$ crash and at least one process in $p_{\Delta+1}, \ldots, p_{2 \Delta}$ does not crash, the decision is obtained in at most 3 rounds. Etc.

It is interesting to observe that, when $m=\ell=k=1$ we have $\Delta=1$ and we obtain a remarkably simple uniform early deciding consensus algorithm for the classical round-based synchronous model $\mathcal{S}_{n, t}[\emptyset]$.

\subsection{Proof and early-decision}

\section{Notation}

- Let $S E N D E R S[r]$ be the set of the processes $p_{i}$ such that $p_{i}$ is a sender during the round $r$ (i.e., $(r-1) \Delta+1 \leq i \leq r \Delta)$.

- Let $E S T[0]$ be the set of proposed values, and $E S T[r]$ be the set of the values contained in the est ${ }_{i}$ local variables of the processes that decide during $r$ or proceed to $r+1$. 


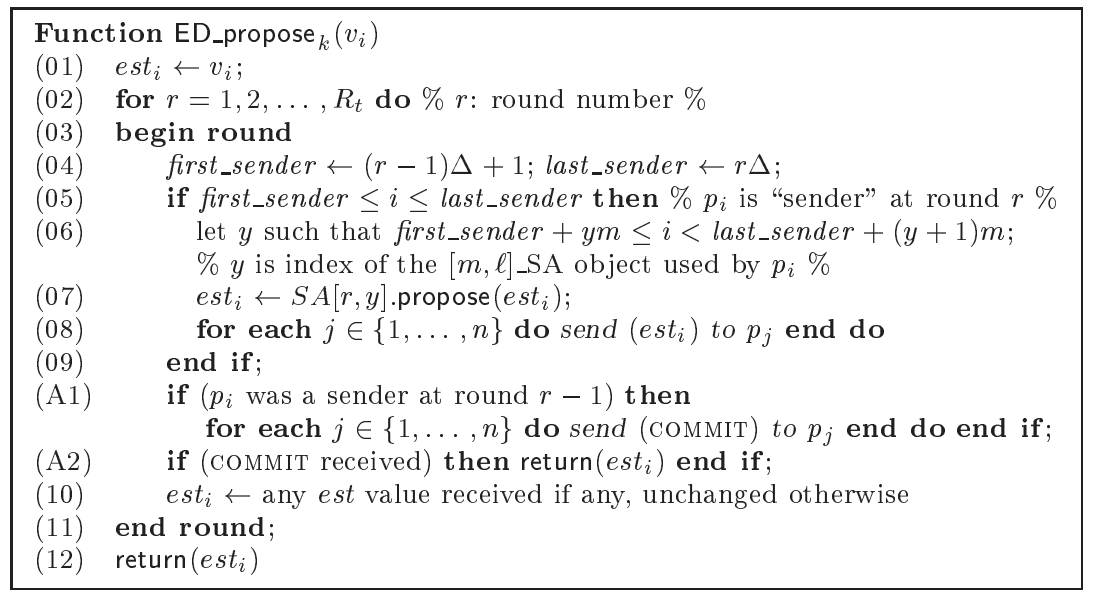

Figure 2: Early-deciding $[n, k]$ _SA object from $[m, \ell]$ _SA objects in a synchronous system (code for $\left.p_{i}\right)$

Lemma 4 At most $k$ different values are decided by the processes.

Proof If no process decides at line A2, the proof is the same as in Lemma 3. So, let us consider the case where at least one process $\left(p_{i}\right)$ decides at line A2. Let us observe that this can occur only after the first round. Let $r$ be the first of these rounds. Let us observe that, until round $r$, we have $E S T[r] \subseteq E S T[r-1]$.

We claim that there are at most $k$ different values present in the system at the end of $r-1$, i.e., $|E S T[r-1]| \leq k$. As $E S T[r] \subseteq E S T[r-1]$, it follows from the claim that there are at most $k$ values in the variables est $t_{x}$ when a process decides. As a value that is decided is a value that is in an est $t_{x}$ variable of a process $p_{x}$ that participates in a round $r^{\prime} \geq r$, it follows that no more than $k$ different values are decided.

Proof of the claim. As $p_{i}$ decides during $r$ at line A2, it received during that round a COMMIT message from some process $p_{j}$ such that $j \in S E N D E R S[r-1]$, from which we conclude that all the processes (that have not crashed) have received, by the end of $r-1$, the value written in est ${ }_{j}$ at line 07 during the round $r-1$. As at most $k$ different values are sent during a round (Lemma 2), it follows that the set EST[r-1] contains at most $k$ different values, which proves the claim and completes the proof of the lemma. $\quad \square_{\text {Lemma } 4}$

Theorem 5 No process executes more than $R_{f}=\min \left(\left\lfloor\frac{f}{\Delta}\right\rfloor+2, R_{t}\right)$ rounds, where $f$ denotes the number of processes that crash during a run.

Proof As for the base algorithm, the proof that no process executes more than $R_{t}$ rounds follows from the code of the algorithm. We have only to show that no process executes more than $\left\lfloor\frac{f}{\Delta}\right\rfloor+2$ rounds.

Let $r_{f}$ be the first round that has a correct sender, i.e., $k \in S E N D E R S\left[r_{f}\right]$. As $f \leq t<n$, it follows that $r_{f} \leq\left\lfloor\frac{f}{\Delta}\right\rfloor+1$. As SENDERS $\left[r_{f}\right]$ contains at least one correct process $p_{k}$, it follows that $p_{k}$ sends a COMMIT message to all the processes during the round $r_{f}+1$ (line A1). This control message is received by each process that has not crashed by the end of $r_{f}+1$, and that process decides accordingly (line A2). It follows that all the processes that execute the round $r_{f}+1 \leq\left\lfloor\frac{f}{\Delta}\right\rfloor+2$ decide and halt by the end of that round.

Theorem 6 The algorithm described in Figure 2 is an early-deciding synchronous t-resilient $k$-set agreement algorithm.

Proof The proof of the validity property is as in Theorem 1. The agreement property has been proved in Lemma 4 . Finally the termination property and the early deciding property have been proved in Theorem 5 . 


\section{Conclusion}

The paper has investigated a new approach to circumvent the $\left\lfloor\frac{t}{k}\right\rfloor+1$ lower bound associated with the $k$-set agreement problem in synchronous systems that can suffer up to $t$ crash failures.

Assuming that the system is composed of clusters of $m$ processes such that the $\ell$-set agreement can be efficiently solved within each cluster (i.e., with a negligible cost with respect to the inter-cluster communication), the paper has shown that it is possible to solve the synchronous $k$-set agreement problem in $R_{t}=\left\lfloor\frac{t}{m\left\lfloor\frac{k}{\ell}\right\rfloor+(k \bmod \ell)}\right\rfloor+1$ rounds (a round being counted as an inter-cluster communication). When considering early decision, it has been shown that a very simple addition of two statements to the base algorithm produces an early deciding algorithm in which no process decides and halts after $R_{f}=\min \left(\left\lfloor\frac{f}{\Delta}\right\rfloor+2,\left\lfloor\frac{t}{\Delta}\right\rfloor+1\right)$ rounds (where $\left.\Delta=m\left\lfloor\frac{k}{\ell}\right\rfloor+(k \bmod \ell)\right), f$ being the actual number of crashes in a run $(0 \leq f \leq t)$.

The paper has also shown that the bound $R_{t}=\left\lfloor\frac{t}{m\left\lfloor\frac{k}{\ell}\right\rfloor+(k \bmod \ell)}\right\rfloor+1$ is a lower bound. This shows an inherent tradeoff relating the "narrowing" power of the base objects that are used and the cost of any $k$-set synchronous agreement algorithm. In that sense, the paper generalizes the previous $R_{t}=\left\lfloor\frac{t}{k}\right\rfloor+1$ lower bound that "implicitly" considers base object without narrowing power. In a very interesting way, this optimality proof relies on two important theorems of distributed computing. One (due to Gafni) is on the number of rounds of a synchronous algorithm that can be simulated in an asynchronous system prone to failures. The second (due to Herlihy and Rajsbaum) states an impossibility on asynchronously solving the $k$-set agreement problem from some base objects. In that sense, the paper shows another link connecting possibility/impossibility results in asynchronous systems and efficiency in synchronous systems.

\section{References}

[1] Aguilera M.K. and Toueg S., A Simple Bivalency Proof that $t$-Resilient Consensus Requires $t+1$ Rounds. Information Processing Letters, 71:155-178, 1999.

[2] Aguilera M.K., Le Lann G. and Toueg S., On the Impact of Fast failure Detectors on Real-Time Fault-Tolerant Systems. Proc. 16th Symposium on Distributed Computing (DISC'02), Springer-Verlag LNCS \#2508, pp. 354369, 2002 .

[3] Attiya H. and Welch J., Distributed Computing, Fundamentals, Simulation and Advanced Topics (Second edition). Wiley Series on Parallel and Distributed Computing, 414 pages, 2004.

[4] Borowsky E. and Gafni E., Generalized FLP Impossibility Results for $t$-Resilient Asynchronous Computations. Proc. 25th ACM Symposium on Theory of Distributed Computing (STOC'93), ACM Press, pp. 91-100, 1993.

[5] Charron-Bost B. and Schiper A., Uniform Consensus is Harder than Consensus. Journal of Algorithms, 51(1):1537, 2004.

[6] Chaudhuri S., More Choices Allow More Faults: Set Consensus Problems in Totally Asynchronous Systems. Information and Computation, 105:132-158, 1993.

[7] Chaudhuri S., Herlihy M., Lynch N. and Tuttle M., Tight Bounds for $k$-Set Agreement. Journal of the ACM, 47(5):912-943, 2000.

[8] Dolev D., Reischuk R. and Strong R., Early Stopping in Byzantine Agreement. Journal of the ACM, 37(4):720741, April 1990.

[9] Fischer M.J., Lynch N.A., A Lower Bound on the Time to Assure Interactive Consistency. Information Processing Letters, 14(4):183-186, 1982.

[10] Fischer M.J., Lynch N.A. and Paterson M.S., Impossibility of Distributed Consensus with One Faulty Process. Journal of the ACM, 32(2):374-382, 1985.

[11] Gafni E., Round-by-round Fault Detectors: Unifying Synchrony and Asynchrony. Proc. 17th ACM Symposium on Principles of Distributed Computing (PODC'00), ACM Press, pp. 143-152, 1998.

[12] Gafni E., Guerraoui R. and Pochon B., From a Static Impossibility to an Adaptive Lower Bound: The Complexity of Early Deciding Set Agreement. Proc. 37th ACM Symposium on Theory of Computing (STOC 2005), ACM Press, pp. 714-722, 2005. 
[13] Herlihy M.P., Wait-Free Synchronization. ACM Transactions on Programming Languages and Systems, 13(1):124-149, 1991.

[14] Herlihy M.P. and Penso L. D., Tight Bounds for $k$-Set Agreement with Limited Scope Accuracy Failure Detectors. Distributed Computing, 18(2): 157-166, 2005.

[15] Herlihy M.P. and Rajsbaum S., Algebraic Spans. Mathematical Structures in Computer Science, 10(4): 549-573, 2000 .

[16] Herlihy M.P. and Shavit N., The Topological Structure of Asynchronous Computability. Journal of the ACM, 46(6):858-923, 1999.

[17] Keidar I. and Rajsbaum S., A Simple Proof of the Uniform Consensus Synchronous Lower Bound. Information Processing Letters, 85:47-52, 2003.

[18] Lamport L. and Fischer M., Byzantine Generals and Transaction Commit Protocols. Unpublished manuscript, 16 pages, April 1982.

[19] Lynch N.A., Distributed Algorithms. Morgan Kaufmann Pub., San Francisco (CA), 872 pages, 1996.

[20] Mostéfaoui A., Rajsbaum S. and Raynal M., Conditions on Input Vectors for Consensus Solvability in Asynchronous Distributed Systems. Journal of the ACM, 50(6):922-954, 2003.

[21] Mostéfaoui A., Rajsbaum S. and Raynal M., The Combined Power of Conditions and Failure Detectors to Solve Asynchronous Set Agreement. Proc. 24th ACM Symposium on Principles of Distributed Computing (PODC'00), ACM Press, pp. 179-188, 2005.

[22] Mostéfaoui A., Rajsbaum S. and Raynal M., Synchronous Condition-Based Consensus. Distributed Computing, $18(5): 325-343,2006$.

[23] Mostéfaoui A. and Raynal M., $k$-Set Agreement with Limited Accuracy Failure Detectors. Proc. 19th ACM Symposium on Principles of Distributed Computing (PODC'00), ACM Press, pp. 143-152, 2000.

[24] Mostéfaoui A. and Raynal M., Randomized Set Agreement. Proc. 13th ACM Symposium on Parallel Algorithms and Architectures (SPAA'01), ACM Press, pp. 291-297, 2001.

[25] Raynal M., Consensus in Synchronous Systems: a Concise Guided Tour. Proc. 9th IEEE Pacific Rim Int'l Symposium on Dependable Computing (PRDC'02), IEEE Computer Press, pp. 221-228, 2002.

[26] Raynal M. and Travers C., Synchronous Set Agreement: a Concise Guided Tour (with open problems). Proc. 12th Int'l IEEE Pacific Rim Dependable Computing Symposium (PRDC'2006), IEEE Computer Press, pp. 267-274, 2006.

[27] Saks M. and Zaharoglou F., Wait-Free $k$-Set Agreement is Impossible: The Topology of Public Knowledge. SIAM Journal on Computing, 29(5):1449-1483, 2000.

[28] Santoro N., Design and Analysis of Distributed Algorithms. Wiley Series on Parallel and Distributed Computing, 589 pages, 2007.

[29] Wang X., Teo Y.M. and Cao J., A Bivalency Proof of the Lower bound for Uniform Consensus. Information Processing Letters, 96:167-174, 2005.

PI ${ }^{\circ} 1836$ 